\title{
Development of Pharyngocutaneous Fistula after Total Laryngectomy: The Predictive Value of C-reactive Protein/Albumin Ratio
}

\author{
Abitter Yücel ${ }^{1, *}$, Hilal Yücel ${ }^{1}$, Fuat Aydemir², Mert Mutaf ${ }^{3}$, Mehmet Akif Eryılmaz³ ${ }^{3}$, Hamdi Arbağ${ }^{3}$
}

\section{ABSTRACT}

Background: We aimed to evaluate whether C-reactive protein(CRP)/ Albumin ratio (CAR) performed in the early postoperative period after total laryngectomy could be a predictive factor for the development of pharyngocutaneous fistula (PCF).

Methods: The files of patients with laryngeal squamous cell carcinoma who underwent total laryngectomy between January 2005 and January 2019 were retrospectively reviewed. Patients were divided into two groups: patients with PCF (PCF group) and without (Non-PCF group). CAR values and risk factors were compared between groups.

Results: The overall incidence of PCF was $23.2 \%$. There was a statistically significant difference between the two groups in terms of CRP and CAR levels $(p=0.001$ ). The CAR value of 27.05 (sensitivity $=75.0 \%$, specificity $68.2 \%$, area under curve (AUC) $=0.742,95 \%$ confidence interval $0.616-0.868$ ) was determined as a cutoff value to describe the development of fistula in the early postoperative period. In multiple linear regression analysis, there was an independent relationship between presence of PCF and previous RT and CAR value.

Conclusions: CAR, performed in the early postoperative period, may be a new and useful marker for predicting PCF after total laryngectomy.

\section{KEYWORDS}

total laryngectomy; pharingocutaneous fistula; crp/albumin ratio

\section{AUTHOR AFFILIATIONS}

${ }^{1}$ Department of Otorhinolaryngology Head and Neck Surgery, Konya Training and Research Hospital, University of Health Sciences, Konya, Turkey

2 Department of Otorhinolaryngology, Kulu State Hospital, Konya, Turkey

${ }^{3}$ Department of Otorhinolaryngology Head and Neck Surgery, Necmettin Erbakan University, Meram Faculty of Medicine, Konya, Turkey

* Corresponding author: Department of Otorhinolaryngology Head and Neck Surgery, University of Health Sciences, Konya Education Research Hospital, Konya, 42080, Turkey; e-mail: abitteryucel@hotmail.com

Received: 9 March 2020

Accepted: 15 July 2020

Published online: 22 December 2020

Acta Medica (Hradec Králové) 2020; 63(4): 159-163

https://doi.org/10.14712/18059694.2020.58

(c) 2020 The Authors. This is an open-access article distributed under the terms of the Creative Commons Attribution License (http://creativecommons.org/licenses/by/4.0), which permits unrestricted use, distribution, and reproduction in any medium, provided the original author and source are credited. 


\section{INTRODUCTION}

Total laryngectomy (TL), still has critical importance in the treatment of laryngeal cancer. Although the number of operations performed for laryngeal and hypopharyngeal cancer has decreased after 1990s due to the recent advances in survival rates with chemotherapy and radiotherapy, TL provides a good local and regional control, especially in advanced stage patients $(1,2)$. The pharyngocutaneous fistula (PCF) can be defined as dissociation of the pharynx mucosa and resulting in salivary leakage to the skin $(3,4)$. PCF is the most common complication after TL and its management requires experience. PCF is the main reason for increased morbidity, delayed initiation of adjuvant therapy, prolonged hospital stay, increased treatment costs, and significantly reduces quality of life (4). Therefore, prevention of PCF or early diagnosis may reduce morbidity and mortality of patients. There may also be disagreements among the authors about the most important risk factors for the occurrence of this complication (5). However, patients can be categorized for PCF risk considering the factors related to the patient, tumor and treatment.

C-reactive protein(CRP)/Albumin Ratio (CAR), has been reported to be a new inflammatory prognostic marker (6). It is stated that CAR is a new independent prognostic factor for total survival and disease-free survival in many cancer types such as larynx, hypopharynx, bladder, hypopharynx and stomach cancer (6-9). In addition, CAR has been reported to be an independent and significant risk factor for postoperative complications at some surgical procedures (10). In this study, we investigated whether CAR performed in the early postoperative period could be a predictive factor for the development of PCF.

\section{MATERIALS AND METHODS}

The files of patients with laryngeal squamous cell carcinoma who underwent TL surgery between January 2005 and January 2019 were retrospectively reviewed. Patients were divided into two groups: patients with PCF (PCF group) and without (Non-PCF group). Age, sex, alcohol and usage of cigarette, additional disease (hypertension, diabetes mellitus, chronic obstructive pulmonary disease), history of preoperative radiotherapy (RT) and tracheotomy, need for blood transfusion, tumor localization (supraglottic, glottic, subglottic), data on the tumor characteristics (T,N,M Staging, AJCC Stage) (11), status of extralaryngeal extension, postoperative hemoglobin $(\mathrm{Hb})$, white blood cell (WBC), neutrophil/lymphocyte ratio (NLR), platelet/ lymphocyte ratio (PLR), CRP, albumin, CAR value and American Society of Anesthesiologists (ASA) classification of patients were recorded. The blood parameters of the patients between the postoperative 2-4 days were considered in this study. The hematologic values were measured with in the first 20 minutes after venous puncture (bloodskeep in potassium EDTA tubes) and then analysed using Sysmex XP-300 (Sysmex Corporation, Japan). The NLR value was calculated by dividing the neutrophil count to the lymphocyte count, and the PLR by dividing the platelet count to the lymphocyte count. CAR was obtained by dividing the serum CRP level (mg/L) by serum albumin (g/dL). TL patients with and without PCF were compared in terms of these parameters.

All surgical procedures were performed by two experienced surgeons or under their supervision. After TL, pharyngeal closure was performed with T-shaped closure technic using $3 / 0$ vicryl (polyglactin) in all cases. Patients who underwent reconstruction with a flap after laryngectomy were excluded from the study. Each patient was fed on the first postoperative day with a nasogastric tube. All patients were fed with the same food supplement in accordance with their weight. In the postoperative period, patients were given intravenous ampicillin sulbactam for 1 week. PCF was diagnosed by revealing the saliva content around the stoma in the postoperative period. Blood values after PCF development were not recorded. None of the patients receiving preoperative $\mathrm{RT}$ treatment received concurrent chemotherapy. Ethical consent was obtained from the ethics committee of local university with the number 2019/1897 for this study.

\section{STATISTICAL ANALYSIS}

Descriptive statistics were used to compare the general characteristics of all participants. Test of Normality, including Kolmogorov-Smirnov and Shapiro-Wilk tests, was used to determine the distribution of data. The data with normal distribution were given as mean \pm standard deviation. Categorical variables were shown as number (n). The comparison of the numerical data between groups was performed with the appropriate test from Independent Samples T test, Mann-Whitney $\mathrm{Q}$ test, ANOVA and Kruskal-Wallis test. Chi-square and Fisher's exact test were used to compare categorical variables. The receiver operating characteristic (ROC) curve analysis was used to determine the sensitivity and specificity values of CAR in determining PCF development after TL. Statistical Package for Social Sciences (SPSS) Windows software (ver. 22; IBM SPSS, Chicago, USA) was used for all statistical analyses. P value less than 0.05 was considered as statistically significant.

\section{RESULTS}

A total of 86 patients were included in this study. There were 20 patients in the PCF group and 66 in the non-PCF group. The overall incidence of PCF was $23.2 \%$. The mean age of the PCF group was 62.5 and the non-PCF group was 57.6. All of the PCF group consisted of male patients and there were 61 male and 5 female in the non-PCF group. There was no significant difference between the two groups in terms of age and gender. Five patients in the PCF group and 2 patients in the non-PCF group received preoperative RT. There was a significant difference between the groups in terms of preoperative RT treatment $(p=0.007)$. There was no significant difference between the two groups in terms of preoperative tracheotomy, need for blood transfusion and additional disease $(p>0.05)$ (Table 1). The patient who underwent TL due to the $\mathrm{T}_{2}$ tumor was operated for recurrence after RT. 
When we evaluated the blood parameters, there was no statistically significant difference between the groups in terms of $\mathrm{Hb}, \mathrm{WBC}, \mathrm{PLR}$ and albumin levels ( $p>0.05$ ). There was a significant difference between the two groups in terms of CRP, NLR and CAR levels $(p=0.001)$. CRP, NLR and CAR levels were significantly higher in the PCF group (Table 1) The CAR value of 27.05 (sensitivity $=75.0 \%$, specificity $68.2 \%$, area under curve (AUC) $=0.742,95 \%$ confidence interval 0.616-0.868) was determined as a cutoff value to describe the development of PCF in the early postoperative period (Figure 1). In other words, patients with CAR $>27.05$ in the early postoperative period had a higher risk of PCF than patients with a CAR $<27.05$.

There was no significant difference between the PCF group and the non-PCF group in terms of tumor location, TNM classification, disease stage, extralaryngeal extension and ASA score $(p>0.05)$ (Table 2). There was a significant difference between the two groups in terms of preoperative RT status and the number of patients who received RT treatment was higher in the PCF group. Additionally, in multiple linear regression analysis, we found an independent relationship between presence of PCF and previous RT (beta: 0.303, p: 0.003) and CAR value (beta: 0.246, p: 0.014) (Table 3).

Tab. 1 Distribution of risk factors according to the patient groups.

\begin{tabular}{|c|c|c|c|}
\hline Parameters & $\begin{array}{l}\text { Patients } \\
\text { with PCF } \\
(n=20) \pm s d\end{array}$ & $\begin{array}{l}\text { Patients } \\
\text { without PCF } \\
(\mathrm{n}=66) \pm \mathrm{sd}\end{array}$ & P value \\
\hline Age & $62.5 \pm 9.9$ & $57.6 \pm 12.0$ & 0.096 \\
\hline Gender (F/M) & $0 / 20$ & $5 / 61$ & 0.257 \\
\hline $\mathrm{BMI}(\mathrm{kg} / \mathrm{m})$ & $22.5 \pm 2.11$ & $22.1 \pm 1.43$ & 0.668 \\
\hline Smoking & 20 & 65 & 0.767 \\
\hline Alcohol & 2 & 0 & 0.052 \\
\hline Previous RT & 5 & 2 & 0.007 \\
\hline $\begin{array}{l}\text { Cardiovascular } \\
\text { Disease }\end{array}$ & 3 & 8 & 0.496 \\
\hline COPD & 4 & 13 & 0.601 \\
\hline $\begin{array}{l}\text { Preoperative } \\
\text { Tracheotomy }\end{array}$ & 2 & 1 & 0.134 \\
\hline Blood Transfusion & 1 & 15 & 0.064 \\
\hline $\mathrm{Hb}(\mathrm{g} / \mathrm{dL})$ & $13.2 \pm 2.0$ & $13.8 \pm 1.8$ & 0.194 \\
\hline $\mathrm{WBC}\left(10^{3} / \mathrm{mm}^{3}\right)$ & $10.2 \pm 2.6$ & $10.5 \pm 4.1$ & 0.631 \\
\hline $\mathrm{CRP}(\mathrm{mg} / \mathrm{l})$ & $111.7 \pm 45.9$ & $71.5 \pm 28.2$ & 0.001 \\
\hline Albumin (g/dL) & $3.1 \pm 0.5$ & $3.1 \pm 0.6$ & 0.576 \\
\hline CAR & $37.2 \pm 19.1$ & $23.1 \pm 9.7$ & 0.001 \\
\hline NLR & $14.9 \pm 5.4$ & $12.3 \pm 7.9$ & 0.013 \\
\hline PLR & $262.4 \pm 89.5$ & $256.1 \pm 169.3$ & 0.132 \\
\hline
\end{tabular}

PCF: Pharyngocutaneous fistula, BMI: Body Mass Index, RT: Radiotherapy, COPD: Chronic Obstructive Pulmonary Disease, Hb: Hemoglobin, WBC: White Blood Cell, CRP: C-reactive Protein, CAR: C-reactive Protein/Albumin Ratio, NLR: Neutrophil/Lymphocyte Ratio, PLR : Platelet / Lymphocyte Ratio
Tab. 2 Distribution of tumor parameters according to the patient groups.

\begin{tabular}{|c|c|c|c|c|}
\hline \multicolumn{2}{|c|}{ Tumor Parameters } & \multirow{2}{*}{$\begin{array}{l}\text { Patients } \\
\text { with PCF } \\
(\mathbf{n}=\mathbf{2 0}) \\
2\end{array}$} & \multirow{2}{*}{$\begin{array}{l}\begin{array}{l}\text { Patients } \\
\text { without PCF } \\
\text { ( } \mathrm{n}=66)\end{array} \\
8\end{array}$} & \multirow[t]{2}{*}{ P value } \\
\hline \multirow{4}{*}{ Site } & Supraglottic & & & \\
\hline & Glottic & 1 & 7 & \\
\hline & Subglottic & 1 & 3 & \\
\hline & Transglottic & 16 & 48 & 0.530 \\
\hline \multirow{3}{*}{$\begin{array}{l}\text { T classifi- } \\
\text { cation }\end{array}$} & T2 & 0 & 1 & \\
\hline & T3 & 9 & 29 & \\
\hline & T4 & 11 & 36 & 0.883 \\
\hline \multirow{4}{*}{$\begin{array}{l}\text { N classifi- } \\
\text { cation }\end{array}$} & NO & 13 & 33 & \\
\hline & N1 & 2 & 16 & \\
\hline & N2 & 1 & 15 & \\
\hline & N3 & 4 & 2 & 0.961 \\
\hline \multirow{2}{*}{$\begin{array}{l}\text { M classifi- } \\
\text { cation }\end{array}$} & MO & 20 & 66 & \\
\hline & M1 & 0 & 0 & 1.000 \\
\hline \multirow{2}{*}{ Stage } & Stage 3 & 9 & 25 & \\
\hline & Stage 4 & 11 & 41 & 0.375 \\
\hline \multicolumn{2}{|c|}{ Extralaringeal spread } & 7 & 29 & 0.329 \\
\hline \multirow{2}{*}{ ASA } & ASA2 & 9 & 11 & \\
\hline & ASA3 & 24 & 42 & 0.329 \\
\hline
\end{tabular}

T: Tumor, N: Node, M: Metastasis, ASA: AmericanSociety of Anesthesiologists

Tab. 3 Multiple linear regresion analysis.

\begin{tabular}{|l|l|l|l|}
\hline $\begin{array}{l}\text { Dependent } \\
\text { variable }\end{array}$ & $\begin{array}{l}\text { Independent } \\
\text { variables }\end{array}$ & $\begin{array}{l}\text { Beta } \\
\text { regression } \\
\text { coefficient }\end{array}$ & P value \\
\hline $\begin{array}{l}\text { Pharyngocutaneous } \\
\text { fistula + }\end{array}$ & RT & 0.303 & $\mathbf{0 . 0 0 3}$ \\
\hline & CAR & 0.246 & $\mathbf{0 . 0 1 4}$ \\
\hline
\end{tabular}

\section{DISCUSSION}

PCF is a major complication that develops after TL and also a challenge for the surgeon and the patient. TL has become increasingly applicable as salvage procedure with the evolution of non-surgical organ protection protocols in the treatment of larynx and hypopharyngeal squamous cell carcinomas (12). Therefore, in future it is inevitable for head and neck surgeons to deal with more risky patients in terms of wound complications and PCF development. However, Suzana et al. (5) reported that the incidence of PCF was between $5 \%$ and $65 \%$ in the 70 s and 80 s, and between $9 \%$ and $25 \%$ in the last decade.

Although many studies have been conducted on PCF development after TL, there is still no consensus at this topic. However, there are many studies conducted to determine the most important risk factors. In a review, the most important risk factors for the development of PCF were preoperative and postoperative $\mathrm{Hb}<12.5 \mathrm{~g} / \mathrm{dL}$, pre and postoperative albumin $<3.7 \mathrm{~g} / \mathrm{L}$, additional diseases, previous RT and chemoradiotherapy, long surgery time, 


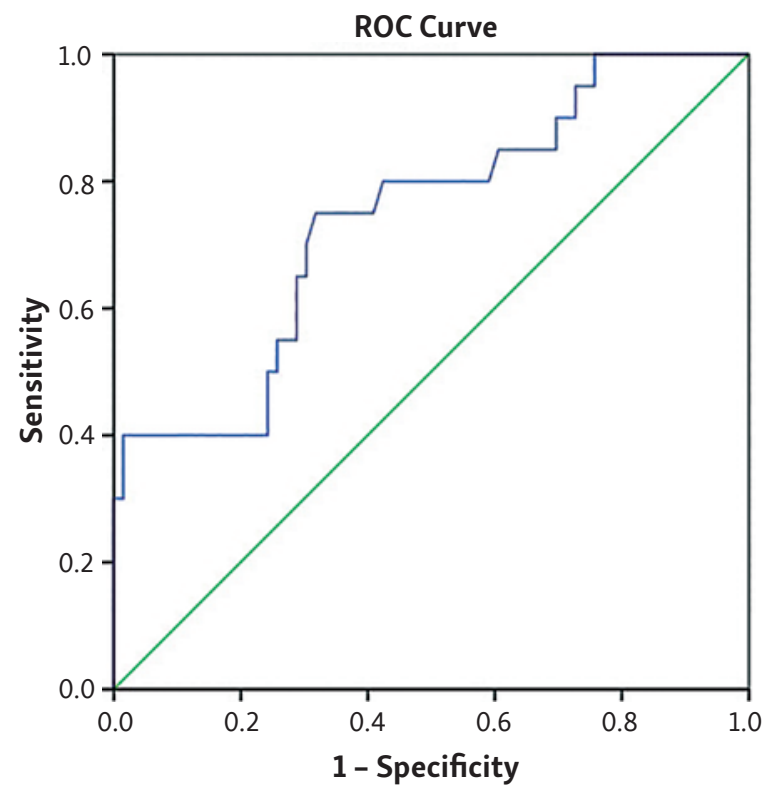

Fig. 1 Reciever operating characteristic (ROC) curve for the development of PCF (sensitivity $=75.0 \%$, specificity $68.2 \%$, area under curve $(A U C)=0.742,95 \%$ confidence interval 0.616-0.868).

blood transfusion during surgery, and inexperience of the surgeon (5). Paydarfar and Birkmeyer (13), reported that postoperative $\mathrm{Hb}<12.5 \mathrm{~g} / \mathrm{dL}$, previous tracheotomy, preoperative RT and preoperative RT combined neck dissection were associated with increased risk of PCF at their meta-analysis. Erdağ et al. (14) reported that control of concurrent systemic disease, preservation of hematological values in the pre- and post-operative period, ensuring adequate nutrition, and preference of erythrocyte suspensions for transfusion are key points in the prevention of PCF development. In addition to these traditionally specified risk factors, it is stated that wound classification, reconstruction with free flap compared to primary closure and ASA classification are among the important risk factors $(15,16)$. Dedivitis et al. (17) reported that, except for the mentioned risks, advanced primary tumor, performance status of patient are the important risk factors.

We evaluated our patients considering the risk factors mentioned above. $23.2 \%$ of our TL patients developed PCF. The main risk factor between the PCF and non-PCF group was previous RT. Although the main significant difference between the two groups was RT, it was observed that smoking was common in both groups, almost all of the patients were in advanced stage and most of the tumors were transglottic. It was previously reported that the absolute risk of PCF development after TL in patients receiving RT for laryngeal SCC mainly depends on the characteristics and localization of the primary tumor. In addition, while in patients with primary glottic $\mathrm{T} 1$ or $\mathrm{T} 2$ tumors, the absolute risk of PCF development was $11 \%$, it was reported to increase to $35 \%$ at T3 or T4 extralaryngeal tumors (18).

In this study, we investigated whether CAR, NLR and PLR could be a predictive factor for early (postoperative 2-4 days) detection of PCF development after TL. Proinflammatory cytokines increase due to surgical injury, leading to changes in circulating acute phase proteins such as
CRP and albumin (19). Postoperative CRP levels are known to be a marker to predict postoperative inflammation and complications (20). Although albumin is associated with chronic diseases and nutritional deficiencies, it behaves like a negative acute phase protein and further decreases in response to surgical stres $(21,22)$. CAR is based on two circulating acute phase protein levels and is associated with surgery-induced inflammation. In addition, CAR is used to identify patients with a high probability of postoperative complications and it is superior to CRP alone in the prediction of postoperative complications (10). Xialong $\mathrm{Ge}$ et al. (10) stated that CAR predicts postoperative results in patients undergoing elective colorectal surgery. They stated that combining CRP and albumin within the single index may be more accurate than CRP alone. In another study, CAR was claimed to be a potential predictor of anastomotic leakage in patients undergoing esophagectomy (23). Aires et al. (24) reported that patients with a NLR value of 2.5 and above had a higher risk of developing postoperative pharyngocutaneous fistula after TL. In our study, while there was a significant difference in NLR values between the two groups, there was no significant difference in terms of PLR. Also, we did not detect any relationship between these two markers and PCF development in the regression analysis.

In this study, CAR was significantly higher in the PCF group than the non-PCF group. We also found that patients with CAR $>27.05$ had a higher risk of PCF than patients with a CAR $<27.05$. Furthermore, there was an independent relationship between presence of PCF and previous $\mathrm{RT}$ and CAR value in multiple linear regression analysis. Perhaps CAR value can give us an idea about need to early compressive dressing or necessary to wait for oral feeding due to the possible PCF. When we examined the groups, we found out that the main difference between the two groups was previous RT. There was no significant difference between the groups in terms of other clinical risk factors. However, serum albumin levels of both groups were below $3.5 \mathrm{~g} / \mathrm{dL}$ which is described as risky level in the literature. The difference between the groups in terms of CAR value was due to the difference in CRP levels rather than the albumin. The role of both albumin and CRP is extensively known in development of PCF. But as we said before, CRP is a positive acute phase reactant and albumin is a negative phase reactant. In the event of a possible PCF, the serum levels of these two markers(CRP, albumin) can change in the opposite direction, therefore combination of these two markers can give more sensitive information about PCF development than the changes alone in the blood levels of these two markers. But probably due to the low number of patients, we could not achieve results that would fully support our hypothesis in this study. Nevertheless, with this study, we wanted to draw attention to the fact that CAR may have a role in the development of PCF after TL and we wanted to inspire the studies that can be carried out with many more patient numbers at this topic.

This study has some limitations. First of all, the retrospective nature of this study may have caused to the exclusion of some patients without detailed medical records. Although the study groups were similar in terms of risk factors in general, there was a difference between 
the groups in terms of previous RT, which is one of the main risk factors of PCF development. Therefore, if this study may be performed in patients with primary TL, more healthy outcomes could be obtained. In addition, only patients who underwent TL with the diagnosis of laryngeal SCC were included in the study, patients with hypopharynx SCC were not included. Another limitation of this study is the lack of CAR values of patients at the preoperative period.

\section{CONCLUSION}

This is the first study to examine the relationship between PCF development and CAR in TL patients. CAR, performed in the early postoperative period, may be a new and useful marker for predicting PCF after TL. However, role of CAR at PCF after TL should be supported by studies with a higher number of patients in order to make more accurate comments on this subject. Especially in high-risk patients, we think that correlating it with other blood parameters can be more useful.

\section{FINANCIAL DISCLOSURE}

The authors declare that this study has received no financial support. This research did not receive any specific grant from funding agencies in the public, commercial, or not-for-profit sectors.

\section{CONFLICT OF INTEREST}

The authors declare that they have no conflict of interest.

This study have been approved by the ethics committee of Necmettin Erbakan University with the number 2019/1897 and have been performed in accordance with the ethical standards as laid down in the 1964 Declaration of Helsinki and its later amendments or comparable ethical standards.

An informed consent was supplied from the participants.

\section{REFERENCES}

1. Hoffman HT, Porter K, Karnell LH, et al. Laryngeal cancer in the United States: changes in demographics, patterns of care, and survival. Laryngoscope 2006; 116 (9 Pt 2 Suppl 111): 1-13.

2. Chu PY, Wang LW, Chang SY. Surgical treatment of squamous cell carcinoma of the hypopharynx: analysis of treatment results, failure patterns, and prognostic factors. J Laryngol Otol 2004; 118(6): 443-9.

3. Makitie AA, Irish J, Gullane PJ. Pharyngocutaneous fistula. Curr Opin Otolaryngol Head Neck Surg 2003; 11(2): 78-84.

4. Mattioli F, Bettini M, Molteni G, et al. Analysis of risk factors for pharyngocutaneous fistula after total laryngectomy with particularfocus on nutritional status. Acta Otorhinolaryngol Ital 2015; 35(4): 243-8.
5. Cecatto SB, Soares MM, Henriques T, Monteiro E, Moura CI. Predictive factors for the postlaryngectomy pharyngocutaneous fistula development: systematic review. Braz J Otorhinolaryngol 2014; 80(2): 167-77.

6. Yu ST, Zhou Z, Cai $Q_{2}$ et al. Prognostic value of the C-reactive protein/ albumin ratio in patients with laryngeal squamous cell carcinoma. Onco Targets Ther 2017; 10: 879-84.

7. Mao $\mathrm{M}$, Wei $\mathrm{X}$, Sheng $\mathrm{H}_{2}$ et al. C-reactive protein/albumin and neutrophil/lymphocyte ratios and their combination predict overall survival in patients with gastric cancer. Oncol Lett 2017; 14(6): 7417-24.

8. Guo Y, Cai K, Mao $\mathrm{S}_{2}$ et al. Preoperative C-reactive protein/albumin ratio is a significant predictor of survival in bladder cancer patients after radical cystectomy : a retrospective study. Cancer Manag Res 2018; 10: 4789-804.

9. Yang X, Liu H, He $\mathrm{M}_{2}$ et al. Prognostic value of pretreatment C-reactive protein/albumin ratio in nasopharyngeal carcinoma: A meta-analysis of published literature. Medicine (Baltimore) 2018; 97(30): e11574.

10. Ge X, Cao Y, Wang $\mathrm{H}_{2}$ et al. Diagnostic accuracy of the postoperative ratio of C-reactive protein to albumin for complications after colorectal surgery. World J Surg Oncol 2017; 15(1): 15 .

11. Edge SB, Compton CC. The American Joint Committee on Cancer: the 7th edition of the AJCC cancer staging manual and the future of TNM. Ann Surg Oncol 2010; 17(6): 1471-4.

12. Busoni M, Deganello A, Gallo O. Pharyngocutaneous fistula following total laryngectomy: analysis of risk factors, prognosis and treatment modalities. Acta Otorhinolaryngol Ital 2015; 35(6): 400-5.

13. Paydarfar JA, Birkmeyer NJ. Complications in head and neck surgery: a meta-analysis of postlaryngectomy pharyngocutaneous fistula. Arch Otolaryngol Head Neck Surg 2006; 132(1): 67-72.

14. Erdag MA, Arslanoglu S, Onal K, Songu M, Tuylu AO. Pharyngocutaneous fistula following total laryngectomy: multivariate analysis of risk factors. Eur Arch Otorhinolaryngol 2013; 270(1): 173-9.

15. Cecatto SB, Monteiro-Soares M, Henriques T, Monteiro E, Moura CI. Derivation of a clinical decision rule for predictive factors for the development of pharyngocutaneous fistula postlaryngectomy. Braz J Otorhinolaryngol 2015; 81(4): 394-401.

16. Lebo NL, Caulley L, Alsaffar H, Corsten MJ, Johnson-Obaseki S. Peri-operative factors predisposing to pharyngocutaneous fistula after total laryngectomy: analysis of a large multi-institunitional patient cohort. J Otolaryngol Head Neck Surg 2017; 23; 46:54.

17. Dedivitis RA, Aires FT, Cernea CR, Brandao LG. Pharyngocutaneous fistula after total laryngectomy: systemic review of risk factors. Head Neck 2015; 37(11): 1691-7.

18. Aarts MC, Rovers MM, Grau C, Grolman W, van der Heijden GJ. Salvage laryngectomy after primary radiotherapy: what are prognostic factors for the development of pharyngocutaneous fistulae? Otolaryngol Head Neck Surg 2011; 144(1): 5-9.

19. Watt DG, McSorley ST, Horgan PG, McMillan DC. Enhanced Recovery After Surgery: Which Components, If Any, Impact on The Systemic Inflammatory Response Following Colorectal Surgery?: A Systematic Review. Medicine (Baltimore) 2015; 94(36): e1286.

20. Warschkow R, Beutner U, Steffen T, Müller SA, Schmeid BM, Güller U. Safe and early discharge after colorectal surgery due to C-reactive protein: a diagnostic meta-analysis of 1832 patients. Ann Surg 2012; 256(2): 245-50.

21. Fairclough E, Cairns E, Hamilton J, Kelly C. Evaluation of a modified early warning system for acute medical admissions and comparisonwith C-reactive protein/albumin ratio as a predictor of patient outcome. Clin Med 2009; $9(1): 30-3$.

22. Sung J, Bochicchio GV, Joshi M, Bochicchio K, Costas A, Tracy K. Admission serum albumin is predicitve of outcome in critically ill trauma patients. Am Surg 2004; 70(2): 1099-102.

23. Shao CY, Liu KC, $\mathrm{Li} \mathrm{CL}_{2}$ et al. C-reactive protein to albumin ratio is a key indicator in a predictive model for anastomosis leakage after esophagectomy: Application of classification and regression tree analysis. Thorac Cancer 2019; 10(4): 728-37.

24. Aires FT, Dedivitis RA, Kulcsar MAV, Ramos DM, Cernea CR. Neutrophil-to-lymphocyte ratio as a prognostic factor for pharyngocutaneous fistula after total laryngectomy. Acta Otorhinolaryngol Ital 2018; $38(1): 31-7$ 